\title{
Optimum speed controller structure utilizing the MCA approach
}

\author{
Khulood Moosa Omran', Basil Hani Jasim², Kadhim H. Hassan ${ }^{3}$ \\ ${ }^{1,2}$ Electrical Engineering Department, Engineering Collage, Basrah University, Basrah, Iraq \\ ${ }^{3}$ Control and automation Department, Basrah Engineering Technical College, Southern Technical University, Iraq
}

\begin{abstract}
Article Info
Article history:

Received Aug 22, 2020

Revised Oct 10, 2020

Accepted Dec 5, 2020

Keywords:

GA approach

MCA approach

Optimal control

Optimization methods

PID control

Speed control

ABSTRACT

In this paper, an optimal speed controller for dc motor is considered using a PID controller and tuned its parameters of gain to offer an optimal solution by using a modified camel algorithm MCA approach. The proposed MCA scheme was applied to solve the difficulty of getting the optimum gains of PID parameters. The MCA has good evolutionary speed with the simple construction of optimization depend on camel searching performance. The characteristics of the MCA algorithm were confirmed by optimizing the gains parameters of proportional, integral, derivative PID controller. The performance of PID-MCA is comparing with a classic PID controller enhanced with GA genetic algorithm optimization method to tune the gain parameters of the speed controller system. It was shown that the utilize of optimization processes indicated better performance for the MCA procedure in term of speed of execution and the size of memory compared with the GA method by applying computer simulations analysis. The proposed scheme has an efficient feature that includes the ease of implementation, good efficiency of computational performances with stable convergence characteristics. The results indicated that the proposed MCA scheme is a useful tool for search ability, produced efficient outcomes compared with the GA optimized method when applied in the proposed system.
\end{abstract}

This is an open access article under the CC BY-SA license.

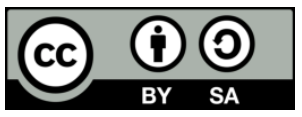

Corresponding Author:

Khulood Moosa Omran

Electrical Engineering Department, Engineering Collage

Basrah University, Basrah, Iraq

Email: khulood.omran@uobasrah.edu.iq

\section{INTRODUCTION}

In preceding years, metaheuristic systems were involved further consideration and brought great attention in researches with optimum results, for instance. The GA approach is the most general evolutionary procedures that are utilized effectively in many engineering applications as the main optimization tool [1]. In common, the algorithms that depend upon intelligence of swarm may be named nature-inspired algorithms such as PSO optimization scheme established by Eberhart et al. [2], which was motivated via the performance of birds' swarm and fish flocking [3]. Various articles compact by optimization schemes motivated depending on animals' performance, for instance, crows, whale, fruit fly, bat and cuckoo search optimization that governed by the different features of animals [4-8]. Later, ant colony optimization ACO technique was established effectively in various fields of engineering applications [9, 10]. A modern algorithm for optimization motivated by the performance of camel travelling which termed camel algorithm CA was proposed in [11]. It is an optimization technique that imitators the activities of the camel when travelling in the wilderness for the searching process to find water resources. The procedure has some weak points such as a complex construction produced by the nesting multi-loop besides several selections for 
parameters that adversely affect the size of memory and the speed of execution. The study in [12] was suggested an intelligent procedure called Camel Herds algorithm (CHA) which governed by the attitude of a camel in the sand, taking into regards, in each herd, there is a leader for every group, the journey for searching food and water reliant on the value of humidity utilizing adjacent strategy. The case study to approve the suggested algorithm was applied for FJSP flexible job of the shop scheduling problem. A modified camel algorithm was proposed in [13] which have a good speed of execution, minor size of memory contrast with previous camel algorithm CA. There are two portions for any proposed metaheuristic procedures which include: the best solutions choice and the random operators. The best solutions choice achieved by converging to the best optimum solution, however the random operators elude trapping the result on local optima besides raising the variety of the results. In this article, the MCA scheme was proposed which is an optimization technique depends on the modified camel travelling behaviour using a simple scheme procedure which is proper to utilize for a widespread of applications.

The control structure of PID is frequently utilized in manufacturing processes since it is easy to achieve, simple and robust [14]. Despite the modest configuration besides robustness of the process, finetuning the optimal parameters of gain in the PID control system is hard relatively [15]. One of the most conventional PID tuning methods is the Ziegler-Nichols method which is the most familiar procedure. For an extensive kind of applied procedures, these tuning method workings reasonably well [16]. Though, occasionally it does not give a good tuning and inclines to give a large overshoot. So, this technique frequently requests returning before used to control manufacturing procedures. Optimization procedures are commonly proposed to tune the control parameters to realize optimal characteristics. The MCA scheme related to the other computational procedures depends on the population. MCA scheme begins with random solutions of the population. MCA scheme is a metaheuristic procedure and its concept develop from utilized the performance of camel searching procedure to get the global optimization. Therefore, the goal of this study is to investigate the MCA scheme to construct a system model for the speed controller and identify optimum parameters of PID gains utilized for the speed controller. The proposed scheme will care about optimum intelligent approaches that constructed tuning the PID of the digital controller using a modified camel algorithm MCA then compared the results with PID-GA scheme using dc motor performances. The rest of this paper is intended as: in section two, the research method of the proposed procedure for PID controller was given which include the genetic optimization approach GA and the principles of the proposed MCA approach. Then section three provides the results and discussion using both proposed algorithms, that includes a contrast among the outcomes acquired utilizing the suggested scheme of MCA-PID with GA-PID scheme through optimal speed controller of dc motor simulation. Our conclusions are indicated in the last section.

\section{RESEARCH METHOD}

In this article, we utilized a modified camel optimization algorithm MCA to design an online selftuning structure of the PID controller to solve the difficulty of getting the optimum gains for the PID parameters. The optimal values of parameters for the PID controller depend on the plant's characteristics. This technique adjusts the PID controller in the loop with the specified system utilizing an optimization procedure. The time-domain of the PID controller is shown [16]:

$$
u(t)=g_{p} E(t)+g_{i} \int E(t) d t+g_{d} \frac{d E(t)}{d t}
$$

where $E(t)$ denotes error variable, that indicates the change between the value of input ref(t) with real output $\mathrm{y}(t) . g_{p}$ is the proportionate gain that will decrease rise time, however, will not decrease the error of steadystate. $g_{i}$ is the gain's controller of integral error which will remove the error of steady-state nevertheless will get a weak transitory response. The derived gain control $g_{d}$ will influence decrease overshoot with developing a transient response. The equivalent model for the structure of the dc motor as indicated in Figure 1. To get a fixed field, the voltage of the armature is needed to be considered. The system model involved a dc motor linked with a load is shown in the following (2) [17]:

$$
\begin{aligned}
& v_{a}(t)=r_{a} i_{a}(t)+L_{a} \frac{d i_{a}}{d t}+e_{b}(t) \\
& e_{b}(t)=k_{b} \cdot w(t) \\
& t_{m}(t)=k_{t} \cdot i_{a}(t)
\end{aligned}
$$




$$
t_{m}(t)=j_{m} \frac{d w(t)}{d t}+b_{m} \cdot w(t)
$$

The armature voltage is $v a(\mathrm{v})$, the armature resistance is $\mathrm{R} a(\Omega)$, the armature inductance is $\mathrm{L} a(\mathrm{H})$, armature current is $i_{a}(\mathrm{~A})$, back emf is $e_{b}(\mathrm{v})$, angular speed is $w(\mathrm{t})(\mathrm{rad} / \mathrm{s})$, the motor torque is $t_{m}(\mathrm{Nm})$, rotor inertia is $j_{m}(K g m 2)$, the viscous friction coefficient is $b m(\mathrm{~N} \mathrm{~ms} / \mathrm{rad})$, torque constant is $k_{t}(\mathrm{Nm} / \mathrm{A})$ and back emf constant is $\mathrm{k}_{b}(\mathrm{vs} / \mathrm{rad})$.

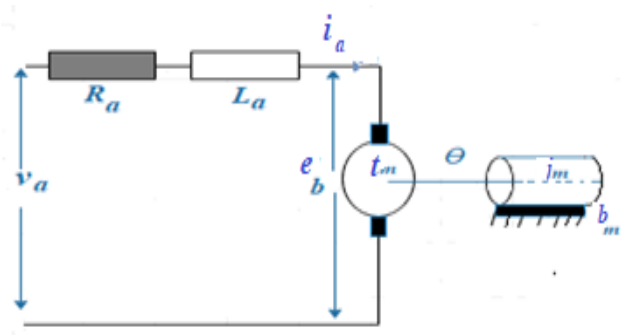

Figure 1. The system model of separable excited dc motor

In Figure 2, the system model of separable excited dc motor was indicated. The system feedback employed as indicated in (2)-(5) which will be analyzed the signal of error between the measured speed and the desired speed then that signal of error is utilized to get the required voltage of command the dc motor. The transfer function between shaft speed and armature voltage at no-load is indicated [18]:

$$
\frac{w(s)}{v_{a}(s)}=\frac{k_{t}}{L_{a} j_{m} s^{2}+\left(R_{a} j_{m}+L_{a} b_{m}\right) s+\left(k_{t} k_{b}+R_{a} b_{m}\right)}
$$

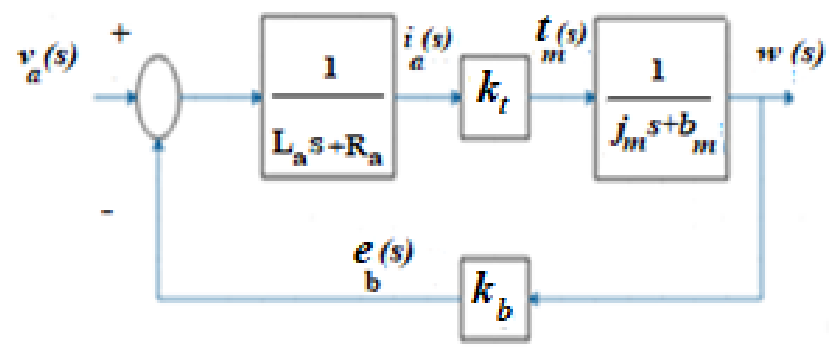

Figure 2. The dc motor block diagram with armature controlled

The motor system model was transformed with two input-one output subsystem. The armature voltage and load torque $T_{\text {load }}$ are input ports and the output ports are angular speed in $w$. For requests when the motor load is to be accelerated or decelerated rapidly, both the electrical time constant and mechanical time constant of the motor shows an essential role. Subsequently, the mechanical time constants of the motor are reducing the rotor inertia. The system model parameters are as proposed in [18]. In the speed controller scheme, the public performance that used were IAE integrated absolute error, ITSE integrated of time weight square Error and ISE integrated of squared error that can be estimated rationally [17]. The drawback of both IAE and ISE performance when they minimized, their influence in the response with rather minor overshoot, with a long settling time. Although ITSE features can overcome the drawback of the ISE features, the derivate procedures of methodical principle are time-consuming and complex. The error feature equations were indicated [18]:

$$
\begin{aligned}
& I A E=\int_{0}^{\infty}\left|r_{r e f}(t)-y(t)\right| d t=\int_{0}^{\infty}|E(t)| d t \\
& I S E=\int_{0}^{\infty} E^{2}(t) d t \\
& \text { ITSE }=\int_{0}^{\infty} t E^{2}(t) d t
\end{aligned}
$$


In this article, a time-domain condition is utilized for estimating the PID controller. Optimal control parameters $g_{p}, g_{i}, g_{d}$ can produce a decent step performance which will affect minimized error response conditions. The response measures include time-domain parameters such as overshoot $\mathrm{Mp}$, the rise time tr, the settling time ts, and the steady-state error Ess. The objective function that is designated to be reduced will be measured to minimize $\mathrm{j}$ is indicated [11]:

$$
j=\left(1-e^{-s}\right)\left(M_{P}+E s s\right)+e^{-s}(t s-t r)
$$

With $g$ is $\left[g_{p}, g_{i}, g_{d}\right]$, and $s$ is the weight element. The fitness function $j$ can fulfill the requirements of designer criteria utilizing the weight issue $s$ value. $s$ utilized to be greater than 0.7 to decrease overshoot with steady states error likewise can use less than 0.7 to decrease rise time and settling time. The optimal choice of $s$ directed on the requirement of a designer and the features of the process that should be controlled. In the design of speed control for dc motor, a minor value of $s$ will lead to additional optimal responses. In our article, using $s$ was fixed to 0.5 for optimal gains of PID control design [13]. In this article, we will utilize the GA algorithm as a traditional tool for tuning the gains of the PID speed controller and compared it with the MCA approach as a proposed method. Genetic algorithm procedure is searched heuristic adaptive algorithm depended on Darwin's evolutionary concepts for genetics and natural selection $[19,20]$. The main idea of genetic algorithms is intended to mimic processes for natural system essential for evolution. The GA maintains the population of individuals, designed for a generation. Each individual signifies a possible solution to the system problem and provides approximately measure of its fitness [21]:

The flow chart of the GA procedure is presented in Figure 3. In each GA cycle, a new generation of possible solutions is produced for a given problem which includes the following steps [22, 23]:

- The initialization: Several distinct results were arbitrarily created initially, using the preliminary population. The aspect of the population is influenced by nature' problem. Conventionally, the population is created arbitrarily, exterior the whole range of possible solutions in the search space.

- The fitness: The strings' performance, frequently called fitness, is then expected by the help of some functions, demonstrating the restrictions of the problem [24].

- The selection: In a genetic algorithm, the operation of selection is the key operation. It is utilized to select the individuals of best fit of the population to produce the new individuals. The new individuals will contribute to additional genetic processes to produce the subsequent generation of the population. The next generation of the population is formed to extend the optimal solution [25].

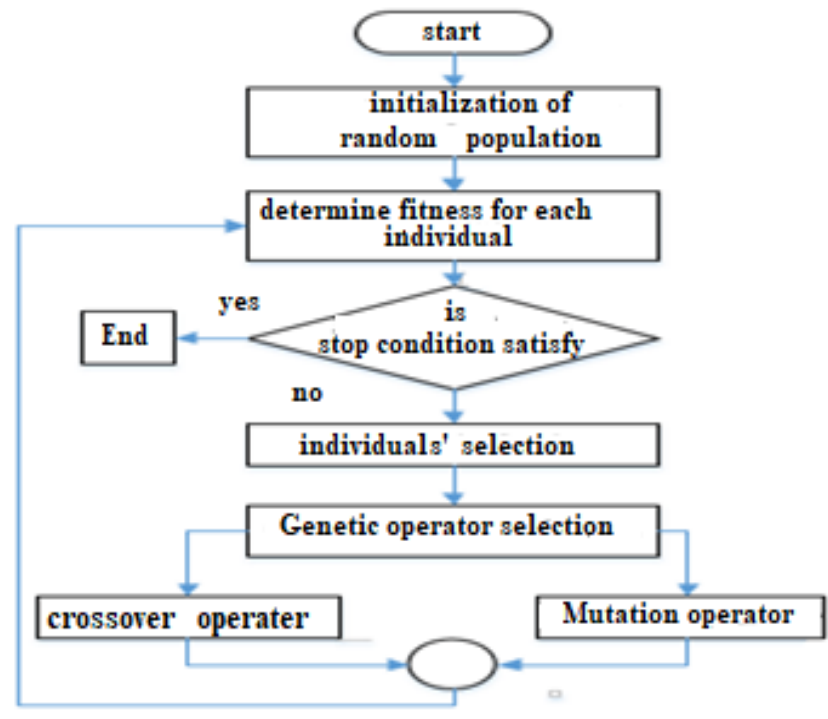

Figure 3. GA flow graph

\subsection{Implementation of modified camel algorithm}

The implementation of modified camel algorithm (MCA) is suitable for resolving problems which having large search space. It achieves fine in problems when the fitness equation is composite, wherever the task is noisy, has a discontinuous, variations along time, and gives various local optima. The range of herd 
search is achieved by just how distant they would move to explore for grass and the source of water. That distance is nearly about a radius of $100 \mathrm{~km}$ [11]. The camel can be able to find out the road with no need of human intrusion, and it could search the places of water by using the sense of smell, following humidity in the air, however, that assumption was not scientifically verified. During travelling procedure to get water, the group's leader supplies associates in the flock to various positions in everywhere to search for water. The camel has a decent sense of path direction so that it can return after a long searching trip [13]. The MCA can discover result solution of space at once in various directions. Therefore, convergence to an optimum solution does not influence on selected initial values of the solution [26]. The proposed method used to improve the performance of the speed controller for the dc motor is the MCA approach. The PID control system is shown in Figure 4, where $v_{a}(t), E(t), w(t)$ are correspondingly reference signal value, error signal variable, and control output variable. The modified camel optimization algorithm is applied to design an online self-tuning structure of the PID controller and to get the optimal values for the gain's controller.

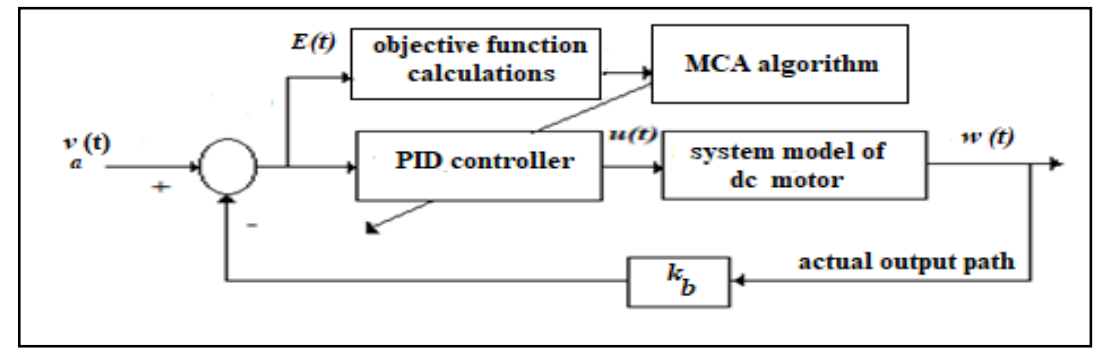

Figure 4. PID-MCA control system

The MCA is stimulated by camel behaviour and roving movement. various aspects are given to summary MCA technique, including the temperature effect, grass and water supply, the camel endurance, the camel vision beside hearing range, the arbitrary walk, and the influence of group effect which may cause multi solutions [11]. We will use tc which refers to temperature, Ec to represent endurance. The influence of temperature was the random element that influenced trip for camel wandering beside it affected on the prospect of a camel. Moreover, each individual will search in contrary regions of the sand which represent the space of searching. It was supposed that there were $\mathrm{n}$ camels in a camel convoy moving over a dimensional location equal to $\mathrm{G}$. The position for camel $i$ in any iteration (itr) of time will be represented using the vector $\mathrm{pc}^{i, i t^{r}}=\left[\mathrm{p}_{1}{ }^{i, i t^{\mathrm{r}}} \mathrm{p}_{2}{ }^{i, i t^{\mathrm{r}}} \ldots \mathrm{p}_{\mathrm{G}}{ }^{i, i t \mathrm{r}}\right]$, where $i=1,2 \ldots, \mathrm{n}$ and $i t \mathrm{r}=1,2, \ldots, i t \mathrm{r}_{\text {max }}$. At the start itr will be 0 , the groups of camels are extended over the sand searching arbitrarily for the water source as assumed in the following [13]:

$$
p c^{i, i t r}=\left(p_{\max }-p_{\min }\right) r n+p_{\min }
$$

Therefore, the position of every camel was exposed toward definite fitness function to conclude the finest position. With $\mathrm{c}$ is $1,2, \ldots, \mathrm{G}, \mathrm{rn}$ represents a random number consistently spread in the range of 0 and $1, p_{\min }$ signifies the minimum bound for the position, $p_{\max }$ represents the maximum bound of the camel position $p_{\min }, p_{\max }$ are considered as optimization variables. The procedure of MCA was represented in Figure 5. The temperature of position straight affects the camel roving, and it changes the camels' endurance considerably. Subsequently, a various camel transfer near sparse places, they meet the different temperature quantity causing various level of endurance for every individual. The temperature value is $t c$ for the camel $i$ in the iteration $i t r$ is variant among min quantity of temperature $t_{\min }$ and max quantity of temperature $t_{\max }$ was denoted:

$$
t c^{i, i t r}=\left(t_{\max }-t_{\min }\right) r n+t_{\min }
$$

The influence of the temperature value on each camel robustness is represented:

$$
E c^{i, i t r}=1-\frac{\left(t c^{i, i t r}-t_{\min }\right)}{t_{\max }-t_{\min }}
$$


The camel endurance is proportional inversely to the temperature as indicated in (13). The upper value of the endurance effects the camel steps to be wider. The hills in the sand would remove the view of some camels in the herd, therefore, they are unable to adjust the track near the food region specified by an assured camel. Two stages for updating the place of each camel are achieved, when the camel view $v$ is greater than the threshold level of vision, the adjust function has been expected as [13]:

$$
p c^{i, i t r}=p c^{i, i t r-1}+E c^{i, i t r}\left(p c^{b e s t}-p c^{i, i t r-1}\right)
$$

where $p c$ best indicated the best position for the total previous repetitions global finest value created by the fitness function. The next state for modifying operation occurs when the camel's vision $v$ is fewer than the threshold vision so that the camel arbitrarily modifies its position subsequent (11).

New positions were exposed to the function of fitness to calculate if the best position of the current trial is better than the previous position. When the current position is superior to the previous trail, formerly the new individual is a global finest position, else previous one stays the global finest position. An essential object would be informed, the camel vision $v$ (at every trial) must be the number of a random value between $(0,1)$ since it will be determined on the existence of the arbitrary positions of the sandhills. The modified operation remains until the maximum trail is done or a definite level of the threshold for fitness function was achieved.

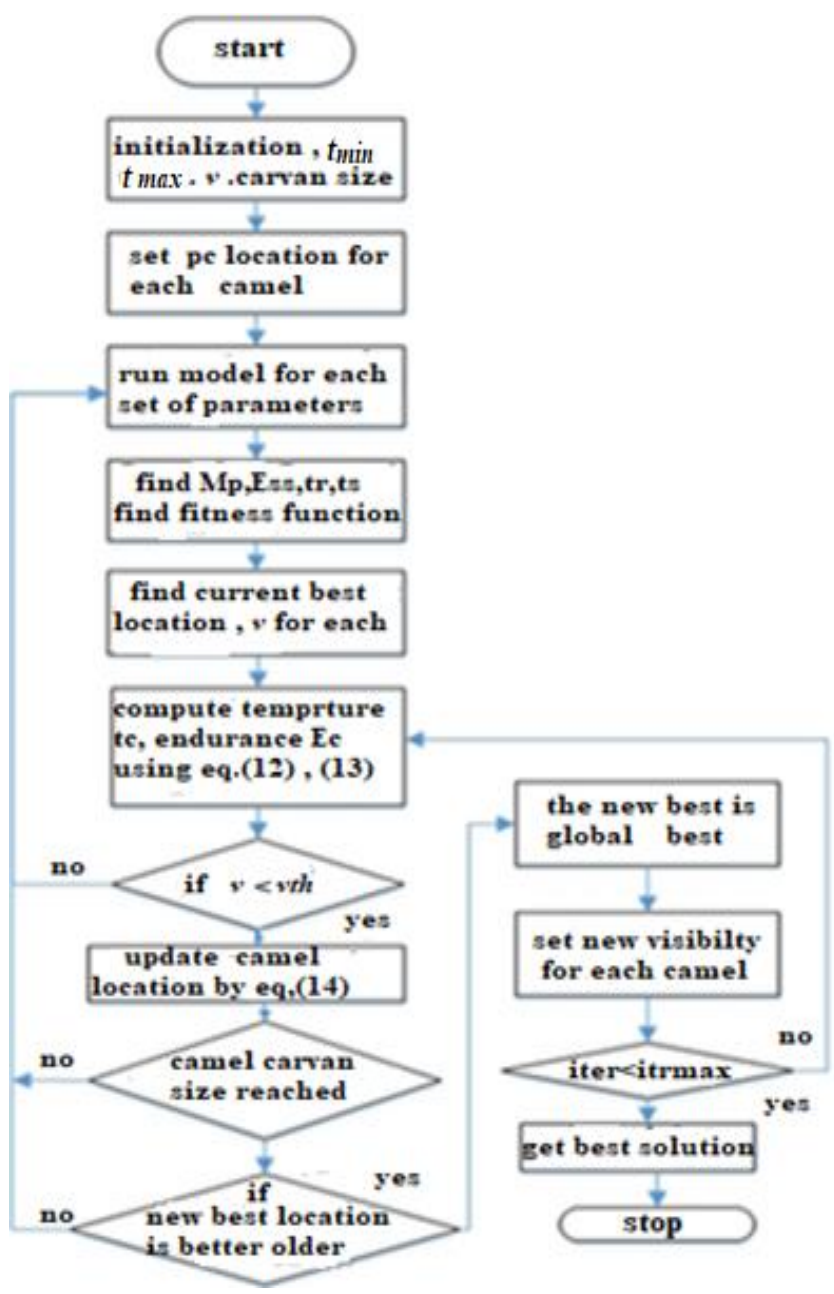

Figure 5. General flow charts of the MCA algorithm

\section{RESULTS AND DISCUSSION}

The m-files in Mat lab are utilized to determine the ability of the modified camel algorithm MCA and compared with the GA algorithm. In the experiments' trails, the iterations number is selected as similar 
for each algorithm to evaluate the performance besides the convergence of speed that acquires the best fitness. The design of $g_{p}, g_{i}$, and $g_{d}$ is carried out assuming that there is no variation in desired speed, the gain' values of $g_{p}, g_{i}$, and $g_{d}$ achieved by the MCA are utilized to show the response of the system while there was a step variation in desired speed in the first iteration. The speed control of the dc motor for 1000 rpm signified a value equal to one-volt signal, conferring to the simulation, the subsequent parameters for optimal MCA are utilized to validate the characteristics of the PID controller enhanced with MCA parameters: the total journey steps are 100 , the initial endurance is 1 , the visibility is 0.1 , the camel caravan is 50, and the dying rate equal zero. However, the experimental parameters that setting for GA was as: the population size is 100 , the crossover rate is 0.9 using one-point crossover type, the mutation rate is 0.05 with one-point mutation type, and by using Roulette wheel selection method. Table 1 indicates the comparison results for the proposed two algorithms with the best fitness. It can be noticed that the MCA reaches to the best fit within the 100 iterations and gives better results than the GA algorithm. The table indicates the characteristics of the PID controller with the optimal three constants. The MCA reaches to a near-global minimum for the test function and gives good results compared with GA. The speed of convergence to the global minimum is an important measure for evaluating the algorithms. It can be concluded from this table that the maximum overshoot and settling time is fine within the restricted boundaries. By association with GA optimal methods, it shows that this technique can increase the dynamic performance of the system in a better way. The main objective functions to be reduced, and it abbreviated the steady-state error, minimized the rise time, minimized the maximum overshoot, minimized the settling time, and the ISE which is denoted the cost function of the plant system.

\begin{tabular}{ccc} 
Table 1. The parameters of proposed PID control scheme \\
\hline Parameters of simulation & PID-GA & PID-MCA \\
\hline$g_{p}$ & 4.157 & 4.58 \\
$g_{i}$ & 0.784 & 0.816 \\
$g_{d}$ & 0.01 & 0.0115 \\
$t r$ & $8.09 \mathrm{~m} \mathrm{sec}$ & $8.6 \mathrm{~m} \mathrm{sec}$ \\
$t s$ & $57.47 \mathrm{~m} \mathrm{sec}$ & $40.02 \mathrm{~m} \mathrm{sec}$ \\
$M p \%$ & 20.28 & 3.3909 \\
Peak time & $20 \mathrm{~m} \mathrm{sec}$ & $20 \mathrm{~m} \mathrm{sec}$ \\
Ess & 0 & 0 \\
Simulation time & $26.2 \mathrm{sec}$ & $8.758 \mathrm{sec}$ \\
ISE & 0.431 & 0.3925 \\
\hline
\end{tabular}

The convergence graphs of a fitness function for the speed controller for each scheme are indicated in Figure 6(a) and (b) which show that PID-MCA gives better performance compared with PID-GA scheme. In Figure 7 the step response for dc motor established with PID speed controller using both proposed optimal techniques are indicated. To offer a comparison which indicates the influence of optimal controller, Figure 7(a) was given to associate the response of the feedback system with PID-GA and Figure 7(b) PID-MCA controller with a step input.

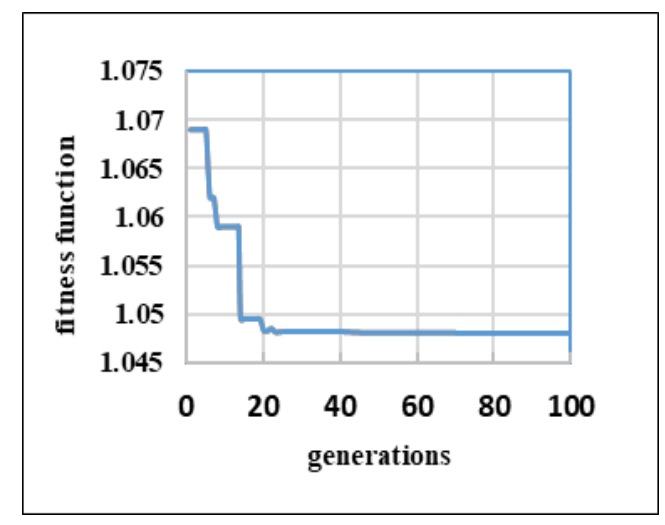

(a)

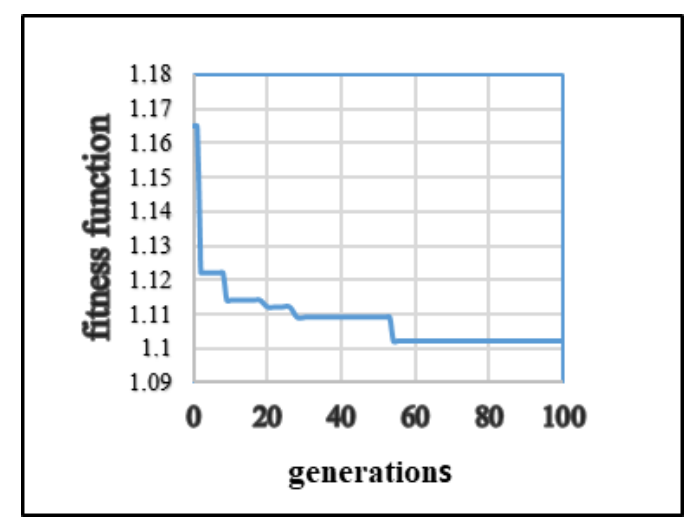

(b)

Figure 6. The convergence of fitness function for speed controller, (a) PID-GA speed controller, (b) PID-MCA speed controller 


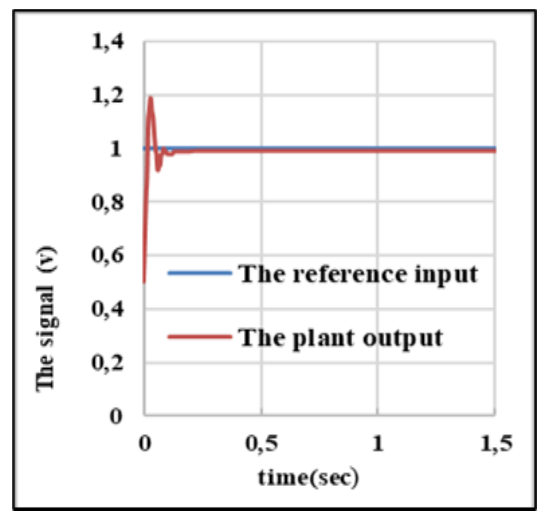

(a)

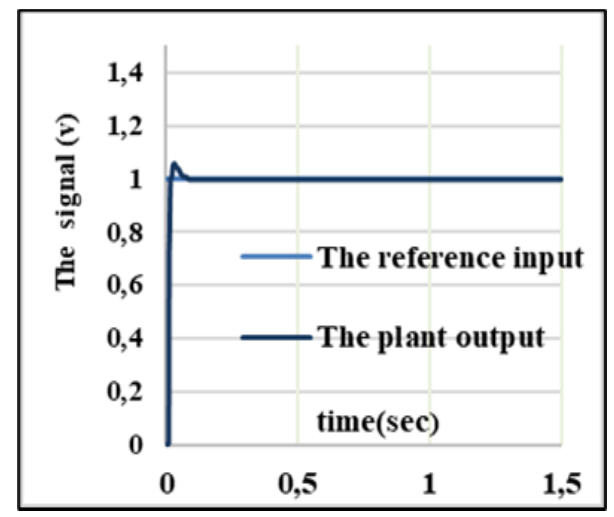

(b)

Figure 7. The step response of dc motor using speed controller, (a) PID-GA, (b) PID-MCA

In Figure 8, the change of the reference input response of system utilizing GA and MCA for the PID controller is shown. The response of the system in the case of variation in the required input where the input signal is varying to the new value at 0.5 seconds then return to the first input value. The results indicated the faster performance of PID-MCA technique compared with PID-GA optimization technique. The MCA scheme can achieve an effective search for the optimum PID controller gains.

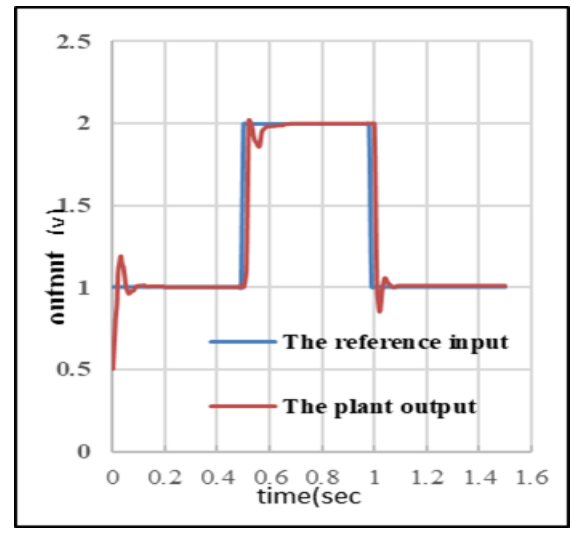

(a)

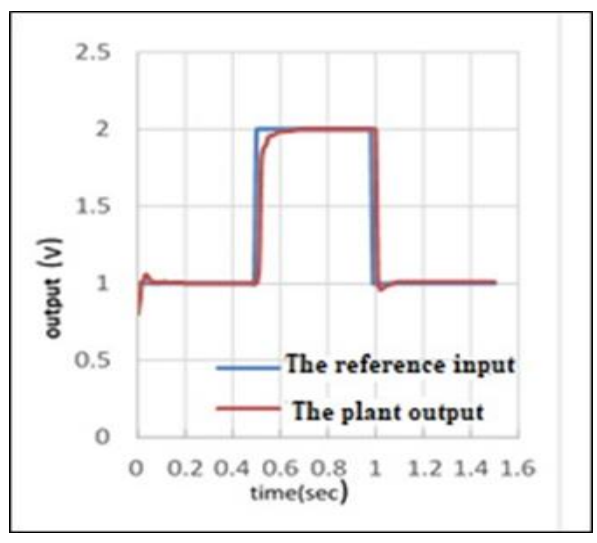

(b)

Figure 8. The change of the reference input response of system utilizing PID controller, (a) PID-GA,

(b) PID-MCA

\section{CONCLUSION}

To solve the difficulty of getting the optimum gains of PID parameters, this article applied a modified procedure of PID-MCA controller and tunes the gain parameters of the speed controller of the dc motor. The MCA scheme has a good speed of calculations with a simplified structure of optimization considered on the behaviour of camel travelling. In term of execution speed and memory size, the results show that the modified camel algorithm MCA is superior to the GA method. It has a high execution speed and small memory size compared with the GA technique for the controller design of a separately excited dc motor. The results of simulations illustrate that the proposed optimal controller can achieve an effective search for the optimum PID controller gains. Furthermore, it was found that the MCA scheme was a good performance for the online optimization schemes that need small size of memory with a fast output response. The simulation results indicated that the optimized speed system has good performance for the optimum PID-MCA controller compared with traditional PID-GA optimal controller. The suggested scheme was more proficient in developing stability of speed performance, with less steady-state error, also it was indicated that the change in the desired input signal did not affect the driving motor characteristics. 


\section{REFERENCES}

[1] Y. Mitsukura, T. Yamamoto, and M. Kaneda, "A design of self-tuning PID controllers using a genetic algorithm," Proc. Amer. Contr. Conf., San Diego, CA, vol. 2, pp.1361-1365, June, 1999.

[2] J. Kennedy and R. Eberhart, "Particle swarm optimization," Proc. IEEE Int. Conf. on Neural Networks, 1995 , vol. 4, pp. 1942-1948.

[3] M. Kumar, B. Das, P. Nallagownden, I. Elamvazuthi, and S. A. Khan, "Optimal configuration of wind farms in radial distribution system using particle swarm optimization technique," Bulletin of Electrical Engineering and Informatics BEEI, vol. 7, no. 2, pp. 286-293, June 2018.

[4] A. Askarzadeh, "A novel metaheuristic method for solving constrained engineering optimization problems: crow search algorithm," Computers and Structures, Elsevier, vol. 169, pp.1-12, 2016.

[5] M. N. Morshidi, I. Musirin, A. R.S. Rahim, M. R. Adzman, and M. H. Hussain, "Whale optimization algorithm based technique for distributed generation installation in distribution system," Bulletin of Electrical Engineering and Informatics BEEI, vol. 7, no. 3, pp. 442-449, Sept. 2018.

[6] K. Omran, "The routing control in mobile ad hoc network using intelligent optimization algorithms," Proc. of the $2^{\text {nd }}$ International Conference on Electrical, Communication and Computer Engineering (ICECCE), Istanbul, Turkey, pp.315-320, 2020.

[7] S. Srivastava and K. Sahana, "Application of bat algorithm for transport network design," J. Computational Intelligence and Soft Computing, vol. 2019, pp.1-12, 2019.

[8] J. Wang, B. Zhou, and S. Zhou, "An improved cuckoo search optimization algorithm for the problem of chaotic systems parameter estimation," Hindawi Publishing Corporation Computational Intelligence and Neuro Science, vol. 2016, pp.1-8, 2016.

[9] V. G. Batista, C. T. Scarpin, J. E. Pécora, and A. Ruiz, "A new ant colony optimization algorithm to solve the periodic capacitated arc routing problem with continuous moves," Mathematical Problems in Engineering, Hindawi, vol. 2019, pp. 1-12, 2019.

[10] R. Jovanovic, M. Tuba, and S. Voss, "An ant colony optimization algorithm for partitioning graphs with supply and demand," Appl. Soft Comput., vol. 41, pp. 317-330, 2016.

[11] M. K. Ibrahim and R. S. Ali, "Novel optimization algorithm inspired by camel traveling behavior," Iraq J. Electrical and Electronic Engineering, vol. 12, no. 2, pp 167-177, 2016.

[12] A. T. Al-Obaidi1, H. S. Abdullah, and Z. O. Ahmed, "Camel Herds algorithm: a new swarm intelligent algorithm to solve optimization problems," International Journal on Perceptive and Cognitive Computing (IJPCC), vol. 3, no. 1, pp. 6-10, 2017.

[13] S. R. Ali, F. M. Alnahwi, and S. A. Abdullah, "A modified camel travelling behavior algorithm for engineering applications," Australian Journal of Electronics Engineering, vol. 16, no. 3, pp. 176-186, 2019.

[14] I. Juniku and P. Marango, "PID design with bio-inspired intelligent algorithms for high order systems," International Journal of Mathematics and Computers in Simulation, vol. 9, pp.44-52, 2015.

[15] C. C. Wong, S. A. Li, and H. Y. Wang, "Optimal PID controller design for AVR system," Tamkang Journal of Science and Engineering, vol. 12, no. 3, pp. 259-270, 2009.

[16] H. S Jain, A. Palak, S. Agrawal, K. Tibrewal, and M. K. Hota, "DC motor speed control using PID controller, IR sensor and PWMh," IJITEE Journal, vol. 8, no. 6, pp. 781-786, 2019.

[17] I. Fatah, "PSO-based tuning of PID controller for speed control of DC motor," Diyala Journal of Engineering Sciences, vol. 7, no. 3, pp. 65-79, September 2014.

[18] S. N. J. Amlashi, "Design and implementation of fuzzy position control system for tracking applications and performance comparison with conventional PID," IAES International Journal of Artificial Intelligence (IJ-AI), vol. 1, no. 1, pp. 31-44, March 2012.

[19] H A. Lafta and A M. Al-Salih, "Efficient routing protocol in the mobile ad-hoc network (MANET) by using genetic algorithm (GA)," IOSR Journal of Computer Engineering (IOSR-JCE), vol. 1, no. 16, pp. 47-54, 2014.

[20] M. Moza and S. Kumar, "Routing in networks using genetic algorithm," Bulletin of Electrical Engineering and Informatics BEEI, vol. 6, no. 1, pp. 88-98, March 2017.

[21] S. Harun and M. F. Ibrahim, "A genetic algorithm-based task scheduling system for logistics service robots," Bulletin of Electrical Engineering and Informatics BEEI, vol. 8, no. 1, pp. 206-213, March 2019.

[22] J. Wang, M. Zhang, O. K. Ersoy, K. Sun, and Y. Bi, "An improved real-coded genetic algorithm using the heuristical normal distribution and direction-based crossover," Computational Intelligence and Neuroscience vol. 2019, pp.1-17, 2019.

[23] Y. Wijesinghe and M. Narayana, "Genetic algorithm tuned PID controllers for multivariable level control system," Moratuwa Engineering Research Conference (MERcon), IEEE, Moratuwa, SriLanka, Sri Lanka, pp. 175-180, Jul 2019.

[24] D. C. Meena and A. Devanshu, "Genetic algorithm tuned PID controller for process control," International Conference on Inventive Systems and Control (ICISC), IEEE Coimbatore, pp. 1-6, 2017.

[25] S. N. Anual, M. F. Ibrahim, N. Ibrahim, A. Hussain, M. M. Mustafa, A. B. Huddin, and F. H. Hashim, "GA-based optimisation of a LiDAR feedback autonomous mobile robot navigation system," BEEI Bulletin of Electrical Engineering and informatics, vol. 7, no. 3, pp. 433-441, September 2018.

[26] M. Gebreyohanes and A. Assen, "Adaptation mechanisms of camels (Camelus dromedaries) for desert environment: a review,” Journal of Veterinary Science and Technology, vol. 8, no. 6, pp. 1-5, 2017. 


\section{BIOGRAPHIES OF AUTHORS}
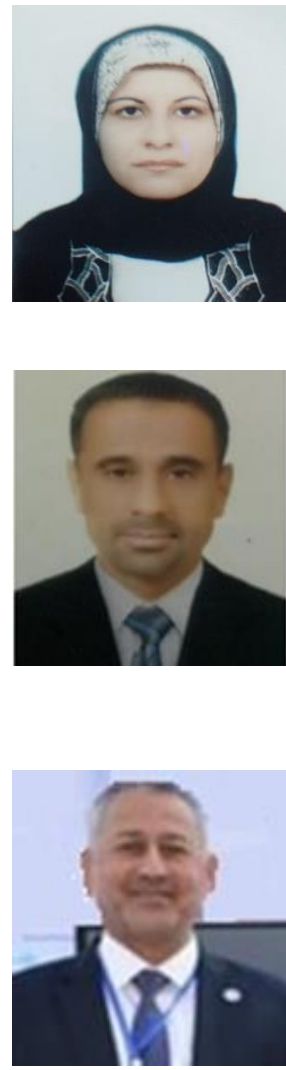

L. Khulood Moosa Omran, she received the B. Sc and M. Sc degrees in Electrical Engineering from the University of Basrah, Basrah, Iraq, she took her Master degree in "Learning control for robot manipulators" in 1997, she worked as a researcher in Arab Gulf studies centre from 20042013, her related work was about the forecasting of electric energy consumption, learning control and Electronic learning, now she is teaching in the electrical engineering department in engineering college, in Basrah University - Iraq. she has more than 18 Published papers in Journals and international conferences in the field of computer and control systems and its applications. Now her related work is optimization, image processing, robot systems and control Systems.

Basil Hani Jasim received the B. Sc and M. Sc degrees in Electrical Engineering and Control \& Computers Engineering from the University of Basrah, Basrah, Iraq in 1995 and 1999 respectively. He also received his $\mathrm{PhD}$ degree in the field of Control and Systems from University of Basrah, Iraq in 2010. He is currently an Assistant Professor at the University of Basrah. His teaching interests covering wide areas of modules across the department of Electrical Engineering, University of Basrah, include Intelligent Control Systems, Robust Control Systems, Microprocessor \& Microcontrollers and Industrial Automation His research interests include Intelligent Control of Robotics, Computational Intelligence, Chaos \& Nonlinear dynamics, Renewable electrical energy systems, and PLC applications in industrial and engineering education.

Kadhim H. Hassan, MSc, PhD in Electrical Engineering, College of Engineering, University of Basra, Iraq. 2000 and 2008 respectively. He joined Basrah Engineering Technical College (BETC) in 2008 as a lecturer. He worked as a Head of Electrical Power Department at (BETC)Southern Technical University for four years, 2013 -2017. He has more than 12 Published papers in Journals and international conferences in the field of intelligent control systems and its applications. 